\title{
Early regression of left ventricular hypertrophy after treatment with esmolol in an experimental rat model of primary hypertension
}

\author{
Begoña Quintana-Villamandos ${ }^{1,2}$, María Jesús Delgado-Martos ${ }^{3}$, Jose Javier Sánchez-Hernández ${ }^{4}$, \\ Jose Juan Gómez de Diego ${ }^{5}$, María del Carmen Fernández-Criado ${ }^{6}$, Fernando Canillas ${ }^{7}$, \\ Antonia Martos-Rodríguez ${ }^{3}$ and Emilio Delgado-Baeza ${ }^{7}$
}

Certain $\beta$-adrenergic blockers have proven useful in the regression of ventricular remodeling when administered as long-term treatment. However, early regression of left ventricular hypertrophy (LVH) has not been reported, following short-term administration of these drugs. We tested the hypothesis that short-term administration of the cardioselective $\beta$-blocker esmolol induces early regression of LVH in spontaneously hypertensive rats (SHR). Fourteen-month-old male SHRs were treated i.v. with vehicle (SHR) or esmolol (SHR-E) $\left(300 \mu \mathrm{gg}^{-1} \mathrm{~min}^{-1}\right)$. Age-matched vehicle-treated male Wistar-Kyoto (WKY) rats served as controls. After $48 \mathrm{~h}$, left ventricular morphology and function were assessed using M-mode echocardiograms (left ventricular mass index (LVMI), ejection fraction and transmitral Doppler (early-to-atrial filling velocity ratio (E/A), E-wave deceleration time (Edec time)). The standardized uptake value (SUV) was applied to evaluate FDG (2-deoxy-2[18F]fluoro-D-glucose) uptake by the heart using PET/CT. Left ventricular subendocardial and subepicardial biopsies were taken to analyze changes in cross-sectional area (CSA) of left ventricular cardiomyocytes and the fibrosis was expressed as collagen volume fraction (CVF). LVMI was lower in SHR-E with respect to SHR $(P=0.009)$. There were no significant differences in $E F, E / A$ ratio or Edec time in SHR-E compared with SHR $\left(P=0.17,0.55\right.$ and $P=0.80$, respectively). PET acquisitions in SHR-E showed lower ${ }^{18} \mathrm{~F}-\mathrm{FDG}$ uptake than SHR $(P=0.003)$. Interestingly, there were no significant differences in SUV in either SHR-E or WKY $(P=0.63)$. CSA in subendocardial and subepicardial regions was minor in SHR-E with respect to SHR $(P<0.001)$, and there were no significant differences in CVF between both groups. Esmolol reverses early LVH in the SHR model of stable compensated ventricular hypertrophy. This is the first study to associate early regression of LVH with administration of a short-term $\beta$-blocker. Hypertension Research (2013) 36, 408-413; doi:10.1038/hr.2012.191; published online 31 January 2013

Keywords: arterial hypertension; echocardiography; esmolol; ventricular hypertrophy

\section{INTRODUCTION}

Left ventricular hypertrophy (LVH) is a strong and independent predictor of cardiovascular morbidity and mortality in patients with hypertension. ${ }^{1}$ Regression of LVH is one of the objectives of antihypertensive therapy and is associated with a reduction in the incidence of cardiovascular events. ${ }^{2}$

Esmolol is an ultrashort-acting cardioselective $\beta$-adrenergic blocker, with a half-life of $\sim 2 \mathrm{~min}$, a time to peak effect of about 6-10 min, and a washout time of $9 \mathrm{~min}^{3}$ Therefore, esmolol is faster acting than other $\beta$-blockers. In addition, the most common adverse effects (hypotension and bradycardia) are quickly reversible. ${ }^{3}$

Treatment to achieve regression of LVH is a priority in clinical practice. The long-term effects of $\beta$-blockers have been shown in both human clinical practice ${ }^{4-6}$ and in experimental animal models. ${ }^{7,8}$
However, early regression of LVH, following short-term use of these agents, has not been analyzed to date.

We tested the hypothesis that short-term administration $(48 \mathrm{~h})$ of esmolol could induce early regression of LVH in 14-month-old spontaneously hypertensive rats (SHRs), using a model of stable compensated LVH. The primary objective was to explore the effect of short-term administration of esmolol on left ventricular mass (LVM). Our secondary objective was to explore cardiac function and metabolism in SHRs.

\section{METHODS}

All procedures conformed to the Guidelines for the Care and Use of Laboratory Animals (NIH publication no. 85-23, revised in 1996) and Spanish

${ }^{1}$ Department of Anesthesiology, Hospital General Universitario Gregorio Marañón, Madrid, Spain; ${ }^{2}$ Department of Pharmacology, Faculty of Medicine Universidad Complutense, Madrid, Spain; ${ }^{3}$ Molecular Biology Laboratory, Department Experimental Medicine and Surgery, Hospital General Universitario Gregorio Marañón, Madrid, Spain; ${ }^{4}$ Department of Preventive Medicine and Public Health, Faculty of Medicine, Universidad Autónoma de Madrid, Madrid, Spain; ${ }^{5}$ Department of Cardiology, Hospital La Paz, Madrid, Spain; ${ }^{6}$ Animal Facility, Universidad Autónoma de Madrid, Madrid, Spain and ${ }^{7}$ Department of Histology, Faculty of Medicine Universidad Autónoma de Madrid, Madrid, Spain Correspondence: Dr B Quintana-Villamandos, Department of Anesthesiology, Hospital General Universitario Gregorio Marañón, Doctor Esquerdo 46, 28007 Madrid, Spain. E-mail: begoquinti@gmail.com

Received 1 April 2012; revised 22 September 2012; accepted 10 October 2012; published online 31 January 2013 
legislation (RD 1201/2005) and were approved by the Ethics Committee of Hospital General Universitario Gregorio Marañón, Madrid, Spain.

\section{Experimental design}

The study animals-14-month-old male SHRs $(n=33)$ and normotensive control Wistar-Kyoto (WKY) rats $(n=12)$-were bred at the animal house of Universidad Autónoma de Madrid. All the rats were supplied with the standard rat chow and drinking water ad libitum and were maintained on a $12 \mathrm{~h} / 12 \mathrm{~h}$ light/dark cycle. The animals were housed at a constant temperature of $24^{\circ} \mathrm{C}$ and relative humidity of $40 \%$. SHRs were randomly divided into two groups (11 rats each): esmolol-treated rats (SHR-E) and vehicle-treated rats (SHR), which was the control group for the SHR-E. Another SHR group (eight rats) was treated with nitroglycerin (SHR-N). All the rats were anesthetized with an i.p. injection of diazepam $4 \mathrm{mg} \mathrm{kg}^{-1}$ and ketamine $10 \mathrm{mg} \mathrm{kg}^{-1}$, and a catheter was inserted into the right internal jugular vein under sterile conditions. The SHR-E received an i.v. infusion of esmolol at $300 \mu \mathrm{g} \mathrm{kg}^{-1}$ $\min ^{-1}$ for $48 \mathrm{~h}$, and the SHR-N an i.v. infusion of nitroglycerin at $0.5 \mathrm{mg} \mathrm{h}^{-1}$ for $48 \mathrm{~h}$. Control SHR and WKY received saline solution (vehicle). Infusion was stopped after $48 \mathrm{~h}$ of treatment and then transthoracic echocardiography was used to study morphology and cardiac function, and PET/CT was applied to study cardiac glucose metabolism. Thereafter, the rat was killed using carbon dioxide, and the hearts were investigated using histological methods.

\section{Blood pressure and heart rate measurements}

Systolic arterial pressure (SAP) and heart rate (HR) were measured (conscious animals prewarmed to $35^{\circ} \mathrm{C}$ in thermostatic cages) by the tail-cuff method with a photoelectric sensor (Niprem 546, Cibertec, Madrid, Spain). Several determinations were made at each session during the treatment $(0,12,24,36$ and $48 \mathrm{~h}$ ), and the findings were considered valid if 10 consecutive measurements were within $10 \mathrm{~mm} \mathrm{Hg}$ of each other.

\section{Echocardiographic studies}

Transthoracic echocardiography was performed using the Acuson Sequoia C512 system (Acuson, Mountain View, CA, USA) equipped with a $13-\mathrm{MHz}$ transducer. The images were acquired with the animals in left lateral decubitus. Transthoracic echocardiography was performed under anesthesia (i.p. diazepam $4 \mathrm{mg} \mathrm{kg}^{-1}$ and ketamine $10 \mathrm{mg} \mathrm{kg}^{-1}$ ) after $48 \mathrm{~h}$ of treatment. Values were determined by averaging the measurements of three consecutive cardiac cycles in accordance with the American Society of Echocardiography guidelines. ${ }^{9}$ M-mode imaging of the parasternal short axis (papillary level) enabled the measurement of LV end systolic and end diastolic internal dimensions (LVIDs and LVIDd, respectively), posterior wall diastolic thickness (PWd) and interventricular septal end diastolic thickness (IVSd). The above measurements were used for calculations of LVM on the basis of the following equation:

$$
\mathrm{LVM}=0.8\left[1.04(\mathrm{IVSd}+\mathrm{LVIDd}+\mathrm{PWd})^{3}-(\text { LVIDd })^{3}\right]+0.6 \mathrm{~g}
$$

LVM was adjusted for body weight by calculating the left ventricular mass index (LVMI). ${ }^{8}$ LV ejection fraction and fractional shortening (FS) were calculated as measures of LV systolic function, as previously described. ${ }^{10}$ In addition, the pulsed-wave Doppler early-to-late transmitral peak diastolic flow velocity ratio (E/A ratio) was measured to assess the diastolic function $(\mathrm{E}$, mitral peak early-filling velocity; and A, mitral peak flow velocity at atrial contraction). The transmitral flow velocity profile was determined by positioning a sample volume at the tip of the mitral valve on the paraapical long-axis view. The E-wave deceleration time was measured as the time interval between peak E-wave velocity and the point where the E-wave descent (or its extrapolation) intercepted the zero line.

Transthoracic echocardiography also enabled the evaluation of relative wall thickness (RWT), calculated as follows: RWT $=$ PWd + IVSd/LVIDd. RWT and $\mathrm{LVM} / \mathrm{BW}$ are used to define the geometric patterns of the LV. ${ }^{11,12}$

\section{Cardiac PET/CT}

PET/CT scans (SEDECAL, Madrid, Spain) were performed in nine rats: WKY $(n=3)$, SHR $(n=3)$, SHR-E $(n=3)$ and SHR-N $(n=3)$. The animals were deprived of food, but allowed free access to water for $8 \mathrm{~h}$ before study. They were anesthetized with isoflurane (3\% induction and $1.5 \%$ for maintenance in $100 \%$ oxygen) using a nose cone. ${ }^{18} \mathrm{~F}$-FDG (2-deoxy-2[18F]fluoro-D-glucose) $[39.37 \pm 3.25 \mathrm{MBq}(1.06 \pm 0.08 \mathrm{mCi})]$ was administered via the venous catheter in the tail vein, over a period of about $15 \mathrm{~s}$, and the catheter was immediately flushed with $100 \mu \mathrm{l}$ of saline. PET scans were performed for $54 \mathrm{~min}$. After PET imaging, the CT scan was acquired for $8 \mathrm{~min}(350 \mu \mathrm{A}, 45 \mathrm{kV}, 200 \mu, 8$ shots and 360 projections).

After images were obtained, the standardized uptake value was used to evaluate FDG uptake by the heart. The standardized uptake value was calculated and normalized according to body weight and the injected dose of FDG. ${ }^{13}$

\section{Morphometric analysis of LV}

After performing the echocardiogram, the heart was excised, weighed and fixed in $4 \%$ paraformaldehyde. Equatorial cross-section of LV was paraffinembedded. Cross-sectional area (CSA) of cardiomyocytes was measured in the subepicardium and subendocardium $(\sim 25$ cardiomyocytes measured in each picture and three pictures per region, for a total of $\sim 150$ cardiomyocytes measured in each LV), as described previously. ${ }^{14}$ Collagen volume fraction was determined as the ratio of collagen surface area with respect to myocardial surface area. ${ }^{15}$

\section{Statistical analysis}

The primary end point was LVM in SHRs after short-term administration of esmolol, which is compared between groups. The variable was expressed as mean \pm s.d. We used the Kolmogorov-Smirnov test to analyze the distribution of quantitative variables. The parameters were compared using repeated measures analysis of variance (physiological parameters) or single-factor (rat) analysis of variance (physiological, echocardiographic and histological parameters). A post hoc Bonferroni's correction was applied. Statistical significance was set at a $P$-value $<0.05$. Statistical analysis was performed using SPSS 17.0 for windows (SPSS, Chicago, IL, USA) and S-PLUS 6.1.

\section{RESULTS}

\section{Physiological parameters}

Values of body weight and blood glucose in WKY, SHR, SHR-E and SHR-N are shown in Table 1. Esmolol lowered SAP in SHR-E with respect to control SHR, and the SAP values during the first $36 \mathrm{~h}$ of treatment with esmolol were comparable to those of the WKY

Table 1 Physiological variables and organ weight of WKY and SHR groups

\begin{tabular}{|c|c|c|c|c|c|}
\hline & $W K Y(\mathrm{n}=9)$ & $S H R(\mathrm{n}=8)$ & $S H R-E(\mathrm{n}=8)$ & $S H R-N(n=8)$ & P-value ANOVA \\
\hline BW (g) & $436.81 \pm 37.34$ & $400.49 \pm 25.84^{*}$ & $400.29 \pm 20.74 *$ & $417.26 \pm 30.30$ & 0.050 \\
\hline HW (g) & $1.51 \pm 0.13$ & $2.07 \pm 0.48^{*}$ & $1.58 \pm 0.14^{\S}$ & $1.93 \pm 0.05^{\ddagger},+\dagger$ & 0.001 \\
\hline Blood glucose $\left(\mathrm{mg} \mathrm{dl}^{-1}\right)$ & $97 \pm 4.24$ & $90 \pm 16.97$ & $93 \pm 4.24$ & $111 \pm 1.15$ & 0.241 \\
\hline
\end{tabular}

Abbreviations: ANOVA, analysis of variance; BW, body weight; HW, heart weight; SHR, spontaneously hypertensive rats treated with vehicle; SHR-E, spontaneously hypertensive rats treated with esmolol; SHR-N, spontaneously hypertensive rats treated with nitroglycerin; WKY, Wistar-Kyoto rats treated with vehicle.

Statistically significant differences between WKY, SHR, SHR-E and SHR-N are shown ( ${ }^{*} P<0.05$ vs. WKY; ${ }^{\ddagger} P<0.001$ vs. WKY; ${ }^{\$} P<0.05$ vs. SHR; $\dagger P<0.01$ vs. SHR-E). Values are given as mean \pm s.d. ANOVA, BW, HW. 
(Figure 1a). Nitroglycerin showed the same level of SAP reduction as the esmolol group. HR remained unchanged in SHR, SHR-N and WKY, but decreased after treatment in SHR-E compared with SHR $(P<0.001)$, SHR-N $(P<0.001)$ and WKY $(P<0.001)$ (Figure $1 \mathrm{~b})$.

\section{Echocardiographic parameters}

Left ventricular geometry. The left ventricular geometry indices obtained from M-mode echocardiography are shown in Table 2. Left


Figure $1 \mathbf{a}$ and $\mathbf{b}$ Changes in systolic arterial pressure (a) and heart rate (b) for Wistar-Kyoto rats treated with vehicle (WKY), spontaneously hypertensive rats treated with vehicle (SHR), spontaneously hypertensive rats treated with nitroglycerin (SHR-N) and spontaneously hypertensive rats treated with esmolol (SHR-E). Data were obtained at 0, 12, 24, 36 and $48 \mathrm{~h}$ of treatment. Values are given as mean \pm s.d. Statistically significant differences are shown: ${ }^{*} P<0.05$ vs. WKY; ${ }^{\dagger} P<0.01$ vs. WKY; $\ddagger P<0.001$ vs. WKY; $\| P<0.01$ vs. SHR; ${ }^{\#} P<0.001$ vs. SHR; $P<0.001$ vs. SHR-E, corrected for multiple comparisons-Bonferroni. ventricular mass (LVM) was significantly higher in control SHR than in WKY $(P<0.01)$. Interestingly, LVM was significantly lower after $48 \mathrm{~h}$ of treatment in SHR-E than in SHR (SHR-E vs. SHR $P<0.01$ ), and it showed no differences with respect to WKY. LVMI was significantly higher in SHR than in WKY $(P<0.001)$. In addition, this indicator of LVH was significantly lower in SHR-E than in SHR (SHR-E vs. SHR $P<0.01$ ). LVMI in SHR-E remained elevated at the limit of statistical significance with respect to WKY $(P=0.05)$. There were no significant differences in LVM and LVMI in SHR-N compared with $\operatorname{SHR}(P=0.37$ and $P=0.73$, respectively); however, either parameters were significantly higher in SHR-N compared with SHR-E (Table 2). SHR presented concentric LVH associated with a significantly higher LVMI and RWT than the WKY $(P<0.001$ and $P<0.001$, respectively). Following treatment with esmolol, both LVMI and RWT were significantly lower in SHR-E compared with SHR $(P<0.01$ and $P<0.001$, respectively). RWT showed no significant differences in SHR-E in comparison with the WKY. Compared with WKY, SHR exhibited a significantly larger IVSd and PW, and a reduced LVIDd and LVIDs. SHR-E showed significantly higher LVIDd and lower IVSd and PW than the SHR treated with vehicle (Figure 2).

Cardiac function. Two indicators of systolic function, FS and ejection fraction, were significantly higher in control SHR than in WKY $(P<0.01$ and $P<0.01$, respectively) (Table 2), thus reflecting the supernormal systolic function of SHR at 14 months of age. Furthermore, there were no significant differences in either functional parameters in SHR-E compared with SHR $(P=0.19$ and $P=0.17$, respectively), neither SHR-E nor SHR compared with SHR-N (Table 2). The data for diastolic function are shown in Table 3. There were no significant differences in the $E / A$ ratio or E-wave deceleration time in SHR and WKY. Although SHR-E showed no significant differences in E-wave deceleration time compared with SHR and WKY, the E/A ratio tended to be higher in SHR-E than in SHR $(P=0.55)$; however, the difference was not statistically significant. There were no significant differences in the E/A ratio or E-wave deceleration time in SHR-N, SHR-E and SHR.

\section{Quantitative analysis of FDG uptake}

The standardized uptake value obtained from the PET study is shown in Figure $3 \mathrm{a}$ and $\mathrm{b}$. SHR showed higher ${ }^{18} \mathrm{~F}$-FDG uptake than WKY $(P<0.01)$. Interestingly, PET acquisitions of the SHR showed lower ${ }^{18} \mathrm{~F}-\mathrm{FDG}$ uptake after $48 \mathrm{~h}$ of treatment with esmolol than SHR treated with vehicle $(P<0.01)$ and SHR treated with nitroglycerin

Table 2 Echocardiographic parameters with M-mode measurements for the WKY and SHR groups

\begin{tabular}{|c|c|c|c|c|c|}
\hline & $W K Y(\mathrm{n}=9)$ & $S H R(\mathrm{n}=8)$ & $S H R-E(\mathrm{n}=8)$ & $S H R-N(\mathrm{n}=8)$ & P-values ANOVA \\
\hline LVIDd (cm) & $0.50 \pm 0.05$ & $0.40 \pm 0.06^{\dagger}$ & $0.57 \pm 0.15^{\|}$ & $0.63 \pm 0.13^{*}, \|$ & 0.002 \\
\hline LVIDs (cm) & $0.22 \pm 0.06$ & $0.10 \pm 0.04 \ddagger$ & $0.22 \pm 0.14$ & $0.16 \pm 0.04^{*}, \S$ & 0.023 \\
\hline IVSd (cm) & $0.27 \pm 0.05$ & $0.40 \pm 0.05^{\ddagger}$ & $0.26 \pm 0.04^{\#}$ & $0.30 \pm 0.01^{\|, * *}$ & $<0.001$ \\
\hline $\mathrm{PWd}(\mathrm{cm})$ & $0.28 \pm 0.06$ & $0.37 \pm 0.05^{\dagger}$ & $0.28 \pm 0.07 \S$ & $0.31 \pm 0.02^{\S}$ & 0.006 \\
\hline LVM (g) & $1.48 \pm 0.25$ & $1.87 \pm 0.20^{\dagger}$ & $1.57 \pm 0.10 \|$ & $2.02 \pm 0.34^{\dagger, * *}$ & $<0.001$ \\
\hline LVMI (mg $\left.{ }^{-1}\right)$ & $3.41 \pm 0.65$ & $4.71 \pm 0.62^{\ddagger}$ & $3.95 \pm 0.33^{*}, \|$ & $4.82 \pm 0.51^{\ddagger}, \dagger \dagger$ & $<0.001$ \\
\hline RWT & $1.13 \pm 0.23$ & $2.00 \pm 0.43^{\ddagger}$ & $1.06 \pm 0.5^{\#}$ & $1.00 \pm 0.20^{\#}$ & $<0.001$ \\
\hline $\mathrm{EF}(\%)$ & $78.64 \pm 9.03$ & $93.08 \pm 4.69^{\dagger}$ & $85.52 \pm 10.93$ & $92.10 \pm 4.86^{\dagger}$ & 0.005 \\
\hline FS (\%) & $55.01 \pm 11.22$ & $74.99 \pm 8.80^{\dagger}$ & $64.51 \pm 14.81$ & $73.31 \pm 9.63^{\dagger}$ & 0.007 \\
\hline
\end{tabular}

Abbreviations: ANOVA, analysis of variance; EF, left ventricular ejection fraction; FS, left ventricular fractional shortening; IVSd, interventricular septal end diastolic thickness; LVIDd, left ventricular end diastolic diameter; LVIDs, left ventricular end systolic diameter; LVM, left ventricular mass; LVMI, left ventricular mass index; PWd, left ventricular posterior wall diastolic thickness; RWT, relative wall thickness; SHR, spontaneously hypertensive rats treated with vehicle; SHR-E, spontaneously hypertensive rats treated with esmolol; SHR-N, spontaneously hypertensive rats treated with nitroglycerin.

Statistically significant differences between WKY, SHR, SHR-E and SHR-N are shown $\left({ }^{*} P<0.05\right.$ vs. WKY; ${ }^{\dagger} P<0.01$ vs. WKY; ${ }^{\ddagger} P<0.001$ vs. WKY; ${ }^{\S} P<0.05$ vs. SHR; $\| P<0.01$ vs. SHR;

${ }^{\#} P<0.001$ vs. SHR; ${ }^{* *} P<0.05$ vs. SHR-E; ${ }^{\dagger} P<0.01$ vs. SHR-E). Values are given as mean \pm s.d. ANOVA, LVIDd, LVIDs, IVSd, PWd, LVM, LVMI, RWT, EF, FS. 



Figure 2 Examples of M-mode echocardiograms from a Wistar-Kyoto rat treated with vehicle (WKY), a spontaneously hypertensive rat treated with vehicle (SHR), a spontaneously hypertensive rats treated with nitroglycerin (SHR-N) and a spontaneously hypertensive rat treated with esmolol (SHR-E) at 14 months of age. IVSd indicates interventricular septal end diastolic thickness and PWd left ventricular posterior wall diastolic thickness. Note that SHR-E showed lower IVSd and PW than SHR.

Table 3 Transmitral inflow measurements for the WKY and SHR groups

\begin{tabular}{|c|c|c|c|c|c|}
\hline & $W K Y(\mathrm{n}=9)$ & $S H R(\mathrm{n}=8)$ & $S H R-E(\mathrm{n}=8)$ & $S H R-N(\mathrm{n}=8)$ & P-value ANOVA \\
\hline E-wave $\left(\mathrm{cms}^{-1}\right)$ & $50.43 \pm 16.90$ & $37.25 \pm 14.86$ & $59.17 \pm 15$ & $44.67 \pm 4.50$ & 0.112 \\
\hline A-wave $\left(\mathrm{cms}^{-1}\right)$ & $29.29 \pm 15.94$ & $33.25 \pm 28.65$ & $39.50 \pm 15.95$ & $30.67 \pm 8.01$ & 0.731 \\
\hline$E / A$ ratio & $1.94 \pm 0.72$ & $1.52 \pm 0.78$ & $1.65 \pm 0.62$ & $1.50 \pm 0.24$ & 0.580 \\
\hline Edec time $\left(\mathrm{ms}^{-1}\right)$ & $42.14 \pm 8.03$ & $43.50 \pm 5.32$ & $42.60 \pm 13.15$ & $35.00 \pm 9.80$ & 0.441 \\
\hline
\end{tabular}

Abbreviations: A-wave, mitral peak filling velocity at atrial contraction; E/A, early-to-atrial filling velocity ratio; Edec time, E-wave deceleration time; SHR, spontaneously hypertensive rats treated with vehicle; SHR-E, spontaneously hypertensive rats treated with esmolol; SHR-N, spontaneously hypertensive rats treated with nitroglycerin; WKY, Wistar-Kyoto rats treated with vehicle.

No significant differences between WKY, SHR, SHR-E and SHR-N. Values are given as mean \pm s.d.

$(P<0.01)$. There were no significant differences in standardized uptake value in either SHR-E or WKY.

\section{Histological parameters}

Value of heart weight (HW) in WKY, SHR, SHR-E and SHR-N is shown in Table 1. CSA of cardiomyocytes in subendocardial and subepicardial regions was minor in SHR-E with respect to SHR $(P<0.001)$, and there were no significant differences in either SHR or SHR-N (Table 4 and Figure 4). Interestingly, SHR-E showed no significant differences in CSA in subendocardial region compared with WKY. There were no significant differences in collagen volume fraction in subendocardial and subepicardial regions between SHR, SHR-N and SHR-E. (Table 4).

\section{DISCUSSION}

Our results show that short-term $(48 \mathrm{~h})$ i.v. infusion of esmolol (300 $\mu \mathrm{g} \mathrm{kg}^{-1} \mathrm{~min}^{-1}$ ) in adult SHR induces early regression of LVH. This is the first study to show an association between early regression of LVH and short-term administration of a $\beta$-blocker. Although previous clinical and experimental animal studies have demonstrated that $\beta$ blockers can reverse LVH, they have all been conducted with drugs administered in the long term. ${ }^{4-8}$

Effects of esmolol on SAP, HR and left ventricular hypertrophy Clinical echocardiographic studies show different patterns in LV geometry in relation to arterial hypertension. LV geometry has been shown to affect both LV function and prognosis. In our study, increasing RWT and LVMI revealed concentric hypertrophy at 14 months of age in the SHR model. This geometric pattern, which indicates the most unfavorable prognosis for hypertensive patients with LV hypertrophy, is considered the typical response to hypertension. . $^{116}$

Our results revealed that esmolol reduced SAP and HR in SHR. This decrease in SAP and HR coincided with less concentric hypertrophy, because LVMI and RWT were lower in SHR after $48 \mathrm{~h}$ of treatment with esmolol than SHR treated with vehicle. In the present study, LVMI in SHR-E remained elevated at the limit of statistical significance with respect to WKY $(P=0.05)$, indicating the transition from concentric to eccentric LV hypertrophy. This transition may have favorable implications for prognosis, in addition to the recognized positive effect of reducing LVMI. ${ }^{17}$ Regression of LVM with antihypertensive therapy (angiotensin-converting enzyme inhibitors, angiotensin receptor blockers, $\beta$-adrenergic blockers, calcium channel blockers and diuretics) has been reported, although only after long-term treatment. ${ }^{18,19}$ In the present study, together with the early reduction of LVM, early changes in CSA of left ventricular cardiomyocytes were observed. Esmolol restored LVM more completely and faster than other antihypertensive drugs (mainly $\beta$-adrenergic blockers). This effect may be attributed in part to the fact that short-term i.v. administration of esmolol exerts a greater hypotensive effect than other $\beta$-blockers (for example, i.v. metoprolol and propranolol at similar degrees of $\beta$-blockade) ${ }^{20}$ This reduction of LVH was produced by a $\beta$ blocker-specific effect (nitroglycerin showed no alterations in LVM, HW, cardiac metabolism or histological parameters).

\section{Effects of esmolol on left ventricular function}

At 14 months of age, SHRs present compensated hypertrophy ${ }^{21}$ with a profile of diastolic dysfunction and concomitant supernormal systolic function, which probably reflects pre-heart failure. Our results are consistent with Cingolani et al., ${ }^{22}$ in which that they confirm the existence of differences in systolic function between adult SHR and WKY (as estimated by ejection fraction and FS). In this context, SHR show enhanced systolic function because of compensated hypertrophy in response to the increased LV wall stress caused by arterial hypertension. $\beta$-Blockade, despite its known negative inotropic effect, has been shown to improve ventricular function when administered in the long term. ${ }^{5}$ Our results showed that there was a trend toward a reduction of hypercontractility in SHR-E. Cardiac $\beta_{1}$-adrenergic receptors are decreased in $\mathrm{SHRs}^{23}$ whereas $\beta_{2}$ adrenergic receptors are unchanged $^{24}$ or even increased. ${ }^{25}$ This reduction in the number of $\beta_{1}$ adrenergic receptors might affect the response of the heart to drugs that act upon myocardial inotropism. ${ }^{26}$ Therefore, the effect of 

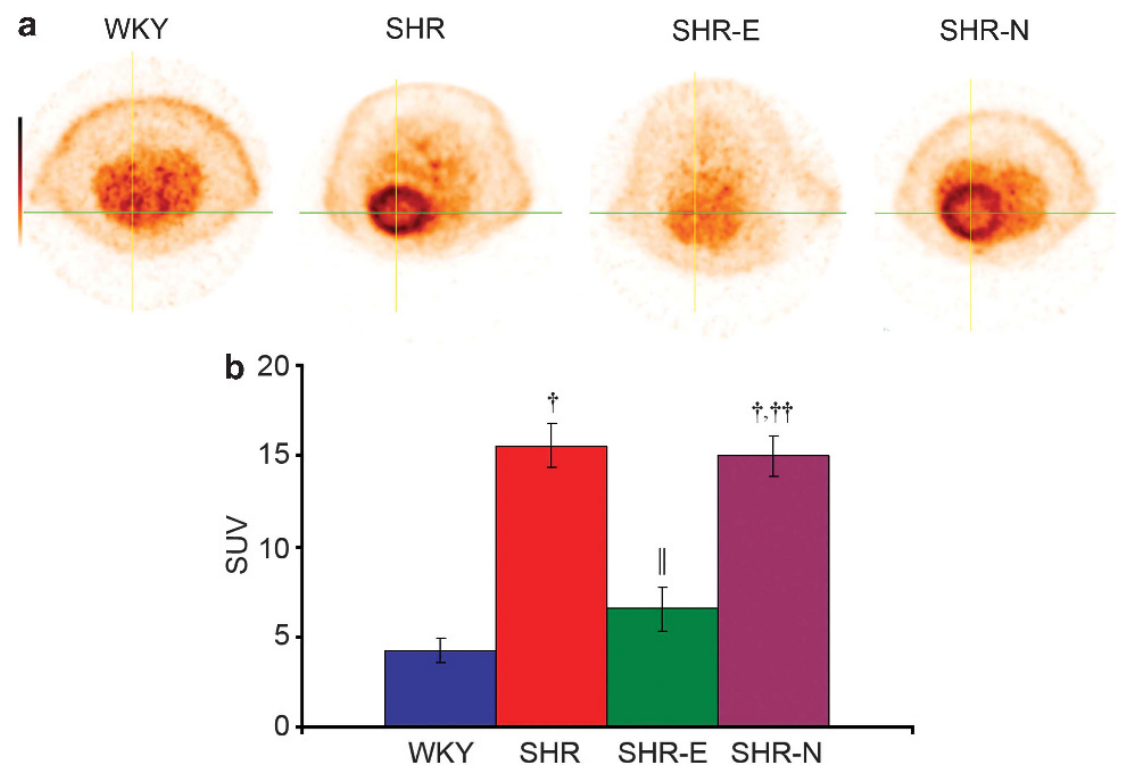

Figure 3 (a, b) PET/CT (PET/CT) images of myocardial 18 F-FDG uptake from a Wistar-Kyoto rat treated with vehicle (WKY), a spontaneously hypertensive rat treated with vehicle (SHR), a spontaneously hypertensive rats treated with nitroglycerin (SHR-N) and a spontaneously hypertensive rat treated with esmolol (SHR-E) (a). There were no significant differences in standardized uptake value (SUV) in either SHR-E $(n=3)$ or WKY $(n=3)$. Values are given as mean \pm s.d. Statistically significant differences are shown: ${ }^{\dagger} P<0.01$ vs. WKY; $\| P<0.01$ vs. SHR; ${ }^{\dagger} P<0.01$ vs. SHR-E, corrected for multiple comparisonsBonferroni (b).

Table 4 Histological variables in two subregions of the LV of WKY and SHR groups

\begin{tabular}{|c|c|c|c|c|c|}
\hline & $W K Y(\mathrm{n}=9)$ & $S H R(\mathrm{n}=8)$ & SHR-E $(\mathrm{n}=8)$ & $S H R-N(\mathrm{n}=8)$ & P-value ANOVA \\
\hline CSA $\left(\mu \mathrm{m}^{2}\right)$ subepicardium & $140 \pm 22$ & $321 \pm 55^{\ddagger}$ & $165 \pm 23^{*}$ & $315 \pm 12^{\ddagger \text {,拉 }}$ & $<0.001$ \\
\hline CSA $\left(\mu \mathrm{m}^{2}\right)$ subendocardium & $139 \pm 21$ & $283 \pm 78^{\ddagger}$ & $142 \pm 11^{\#}$ & 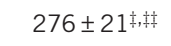 & $<0.001$ \\
\hline CVF (\%) subepicardium & $6.04 \pm 2.69$ & $10.01 \pm 2.68^{*}$ & $11.26 \pm 3.08^{\dagger}$ & $9.03 \pm 2.54^{*}$ & 0.020 \\
\hline CVF (\%) subendocardium & $11.80 \pm 6.69$ & $24.92 \pm 4.68^{\dagger}$ & $24.99 \pm 6.37^{\dagger}$ & $22.80 \pm 3.96^{\dagger}$ & 0.009 \\
\hline
\end{tabular}

Abbreviations: ANOVA, analysis of variance; CSA, cross-sectional area of cardiomyocytes; CVF, collagen volume fraction; SHR, spontaneously hypertensive rats treated with vehicle; SHR-E, spontaneously hypertensive rats treated with esmolol; SHR-N, spontaneously hypertensive rats treated with nitroglycerin; WKY, Wistar-Kyoto rats treated with vehicle Statistically significant differences between WKY, SHR, SHR-N and SHR-E are shown ( ${ }^{\star} P<0.05$ vs. WKY; ${ }^{\dagger} P<0.01$ vs. WKY; ${ }^{\ddagger} P<0.001$ vs. WKY; ${ }^{\sharp} P<0.001$ vs. SHR; ${ }^{\ddagger} P<0.001$ vs. SHR-E). Values are given as mean \pm s.d. ANOVA, CSA, CVF.
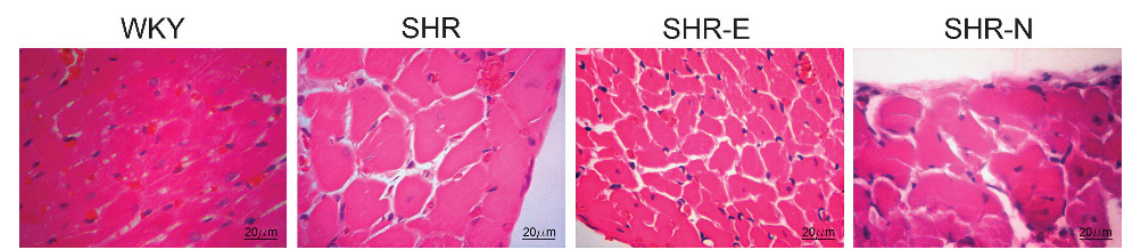

Figure 4 Examples histological sections of subepicardial region of the LV from a Wistar-Kyoto rat treated with vehicle (WKY), a spontaneously hypertensive rat treated with vehicle (SHR), a spontaneously hypertensive rats treated with nitroglycerin (SHR-N) and a spontaneously hypertensive rat treated with esmolol (SHR-E). Note that SHR-E showed lower cross-sectional area of cardiomyocytes than SHR. H\&E 200x.

esmolol ( $\beta_{1}$-blocker) on systolic function could be altered by a decrease in the number of myocardial $\beta_{1}$ receptors.

Our results are consistent with Cingolani et al., ${ }^{22}$ in which they confirm the presence of diastolic dysfunction in adult SHR and WKY. Although some $\beta$-blockers are associated with an improvement in diastolic function, ${ }^{4}$ we found that the administration of esmolol in SHR showed no significant differences in diastolic function with respect to SHR controls. Only studies on human have shown esmolol to lower HR to levels (57-68 beats per $\mathrm{min}$ ) associated with the recovery of diastolic function and we used an experimental animal with an initial HR of about 300 beats per min. ${ }^{27}$

\section{Effects of esmolol on myocardial glucose metabolism}

Energy is generated in the heart from glucose, fatty acids and acetate, although oxidation of fatty acids is the primary source. ${ }^{28}$ Handa et al. ${ }^{29}$ showed a higher rate of glucose uptake in animal models of cardiac hypertrophy. In our study, myocardial glucose metabolism in the SHR model was more marked than in the WKY. These results are 
consistent with those of other authors in this model of LVH, although the animals used were of a different age. ${ }^{28}$ By reducing HR and inotropy, $\beta$-blockers should reduce myocardial energy demands and oxygen consumption, ${ }^{30}$ and the pharmacological effect of $\beta$-blockade in reducing energy demands may thus facilitate biological recovery of myocytes. ${ }^{31}$ This has only been observed after long-term treatment with $\beta$-blockers. Our study showed that short-term treatment with esmolol $(48 \mathrm{~h})$ produced a marked decrease in the glucose metabolism of the hypertrophied ventricle.

In conclusion, our results show that esmolol leads to the regression of cardiac hypertrophy in experimental hypertension, because the esmolol-treated SHR showed lower LVM, lower CSA of cardiomyocytes and lower myocardial glucose metabolism than the control SHR. This is the first study to associate a regression of LVH in a markedly short period of treatment with a $\beta$-blocker. If these results were confirmed in humans, esmolol could be taken into consideration for the treatment of LVH in critical care units.

\section{CONFLICT OF INTEREST}

The authors declare no conflict of interest.

\section{ACKNOWLEDGEMENTS}

This work was supported by a grant from the Spanish Health Ministry (Fondo de Investigaciones Sanitarias) under contract FIS 10/02831.

1 Simko F, Pechanova O. Remodelling of the heart and vessels in experimental hypertension: advances in protection. J Hypertens 2010; 28 (suppl 1): S1-S6.

2 Pierdomenico SD, Cuccurullo F. Risk reduction after regression of echocardiographic left ventricular hypertrophy in hypertension: a meta-analysis. Am J Hypertens 2010; 23: 876-881.

3 Fita G, Gomar C, Rovira I. Esmolol in anesthesiology: pharmacology and indications. Rev Esp Anestesiol Reanim 1999; 46: 401-414.

4 Hurs EM, Zinovieva YA, Poddubnaya AV, Smolenskaya OG. Influence of nebivolol on left ventricular remodeling in patients with arterial hypertension without chronic heart failure. Kardiologiia 2007; 47: 15-19.

5 Devereux RB, Dahlöf B, Gerdts E, Boman K, Nieminen MS, Papademetriou V, Rokkedal J, Harris KE, Edelman JM, Wachtell K. Regression of hypertensive left ventricular hypertrophy by losartan compared with atenolol: the losartan intervention for endpoint reduction in hypertension (LIFE) trial. Circulation 2004; 110: 1456-1462.

6 Colucci WS, Kolias TJ, Adams KF, Armstrong WF, Ghali JK, Gottlieb SS, Greenberg B, Klibaner MI, Kukin ML, Sugg JEREVERT Study Group. Metoprolol reverses left ventricular remodeling in patients with asymptomatic systolic dysfunction: the reversal of ventricular remodeling with Toprol-XL (REVERT) trial. Circulation 2007; 116: 4956

7 Mougenot N, Médiani O, Lechat P. Bisoprolol and hydrochlorothiazide effects on cardiovascular remodeling in spontaneously hypertensive rats. Pharmacol Res 2005; 51: 359-365.

8 Asai T, Kushiro T, Fujita H, Kanmatsuse K. Different effects on inhibition of cardiac hypertrophy in spontaneously hypertensive rats by monotherapy and combination therapy of adrenergic receptor antagonists and/or the angiotensin II Type 1 receptor blocker under comparable blood pressure reduction. Hypertens Res 2005; 28: 79-87.

9 Sahn DJ, DeMaria A, Kisslo J, Weyman A. Recommendations regarding quantitation in M-mode echocardiography: results of a survey of echocardiographic measurements. Circulation 1978; 58: 1072-1083.
10 Loch D, Chan V, Hoey A, Brown L. Rosuvastatin attenuates heart failure and cardiac remodelling in the ageing spontaneously hypertensive rat. Basic Clin Pharmacol Toxicol 2009; 105: 262-270.

11 Ganau A, Devereux RB, Roman MJ, de Simone G, Pickering TG, Saba PS, Vargiu P, Simongini I, Laragh JH. Patterns of left ventricular hypertrophy and geometric remodeling in essential hypertension. J Am Coll Cardiol 1992; 19: $1550-1558$.

12 Kokubo M, Uemura A, Matsubara T, Murohara T. Noninvasive evaluation of the time course of change in cardiac function in spontaneously hypertensive rats by echocardiography. Hypertens Res 2005; 28: 601-609.

13 Graham MM, Peterson LM, Hayward RM. Comparison of simplified quantitative analyses of FDG uptake. Nucl Med Biol 2000; 27: 647-655.

14 Der Sarkissian MarchandEL, Duguay D, Hamet P, deBlois D. Reversal of interstitial fibroblast hyperplasia via apoptosis in hypertensive rat heart with valsartan or enalapril. Cardiovas Res 2003; 57: 775-783.

15 Sevilla MA, Voces F, Carrón R, Guerrero El, Ardanaz N, San Román L, Arévalo MA, Montero MJ. Amlodipine decreases fibrosis and cardiac hypertrophy in spontaneously hypertensive rats: persistent effects after withdrawal. Life Sci 2004; 75: 881-891.

16 Gerdts E, Roman MJ, Palmieri V, Wachtell K, Smith G, Nieminen MS, Dahlöf B, Devreux RB. Impact of age left ventricular hypertrophy regression during antihypertensive treatment with losartan or atenolol (the LIFE study). J Hum Hypertens 2004; 18: 417-422.

17 Cuspidi C, Meani S, Valerio C, Fusi V, Sala C, Maisaidi M, Zanchetti A. Effects of angiotensin II receptor blockade-based therapy with losartan on left ventricular hypertrophy and geometry in previously treated hypertensive patients. Blood Press 2006; 15: 107-115.

18 Fagard RH, Celis H, Thijs L, Wouters S. Regression of left ventricular mass by antihypertensive treatment. A meta-analysis of randomized comparative studies. Hypertension 2009; 54: 1084-1091.

19 Ferreira-Filho C, Abreu LC, Valenti VE, Ferreira M, Meneghini A, Silveira JA, Riera AR, Colombari E, Murad N, Santos-Silva PR, Silva LJ, Vanderlei LC, Carvalho TD, Ferreira C. Anti-hypertensive drugs have different effects on ventricular hypertrophy regression. Clinics 2010; 65: 723-728.

20 Deegan R, Wood MB. $\beta$-Receptor antagonism does not fully explain esmolol-induced hypotension. Clin Pharmacol Ther 1994; 56: 224-228.

21 Brooks WW, Conrad CH, Robinson KG, Colucci WS, Bing OH. L-arginine fails to prevent ventricular remodeling and heart failure in the spontaneously hypertensive rat. $\mathrm{Am} \mathrm{J}$ Hypertens 2009; 22: 228-234.

22 Cingolani $\mathrm{OH}$, Yang XP, Cavasin MA, Carretero OA. Increased systolic performance with diastolic dysfunction in adult spontaneously hypertensive rats. Hypertension 2003; 41: 249-254.

23 Limas C, Limas CJ. Reduced number of $\beta$-adrenergic receptors in the myocardium of spontaneously hypertensive rats. Biochem Biophys Res Commun 1978; 82: 710-714.

24 Yamada S, Ishima T, Tomita T, Hayashi M, Okada T, Hayashi E. Alterations in cardiac alpha and beta adrenoceptors during the development of spontaneous hypertension. J Pharmacol Exp Ther 1984; 228: 454-460.

25 Michel MC, Wang XL, Schlicker E, Göthert M, Beckeringh JJ, Brodde O-E. Increased $\beta_{2}$-adrenoreceptor density in heart, lung and kidney of spontaneously hypertensive rats. J Auton Pharmacol 1987; 7: 41-51.

26 Nordlander MI. Functional consequences of structural adaptation of the heart in hypertension. Hypertension 1984; 6: 58-63.

27 Jurkevicius R, Sakalyte G, Kavoliuniene A. Effects of beta blockers on left ventricular diastolic function: the importance of optimal heart rate reduction. Acta Cardiol 2007; 62: 269-724.

28 Veress AI, Weiss JA, Huesman RH, Reutter BW, Taylor SE, Sitek A, Feng B, Yang Y, Gullberg GT. Measuring regional changes in the diastolic deformation of the left ventricle of SHR rats using microPET technology and hyperelastic warping. Ann Biomed Eng 2008; 36: 1104-1117.

29 Handa N, Magata Y, Mukai T, Nishina T, Konishi J, Komeda M. Quantitative FDG-uptake by positron emission tomography in progressive hypertrophy of rat hearts in vivo. Ann Nucl Med 2007; 21: 569-576.

30 Mueller H, Ayres S. The role of propranolol in the treatment of acute myocardial infarction. Prog Cardiovas Dis 1977; 19: 405-412.

31 Eichhorn EJ, Bristow M. Medical therapy can improve the biological properties of the chronically failing heart: a new era in the treatment of heart failure. Circulation 1996; 94: 2285-2296. 